\title{
Tsafon
}

Revue d'études juives du Nord

$76 \mid 2018$

Expressions yiddish de la nature

\section{Lazar Zamenhof (1859-1917). Du sionisme au hillélisme et de l'hébreu à l'espéranto}

\section{Christian Lavarenne}

\section{(2) OpenEdition}

12 Journals

Édition électronique

URL : https://journals.openedition.org/tsafon/1379

DOI : $10.4000 /$ tsafon. 1379

ISSN : 2609-6420

Éditeur

Association Jean-Marie Delmaire

Édition imprimée

Date de publication : 1 décembre 2018

Pagination : 123-142

ISSN : 1149-6630

\section{Référence électronique}

Christian Lavarenne, «Lazar Zamenhof (1859-1917). Du sionisme au hillélisme et de l'hébreu à

l'espéranto », Tsafon [En ligne], 76 | 2018, mis en ligne le 30 juin 2019, consulté le 25 juin 2021. URL http://journals.openedition.org/tsafon/1379; DOI : https://doi.org/10.4000/tsafon.1379

Tsafon. Revues d'études juives du Nord 


\section{Varia : Histoire}

\section{Lazar Zamenhof (1859-1917) Du sionisme au hillélisme et de l'hébreu à l'espéranto}

\section{Christian Lavarenne*}

L'ophtalmologue juif de Russie Lazar Zamenhof ${ }^{1}$, dont l'année du centenaire de la mort a été clôturée à l'Unesco le 11 décembre 2017 à l'initiative de la Pologne ${ }^{2}$, n'a pas été seulement l'inventeur maintenant reconnu de l'espéranto mais aussi l'initiateur, peu suivi car incompris, d'une «organisation socio-religieuse ${ }^{3}$ au sein du judaïsme, devant apporter la «solution de la question juive », but fondamental de sa vie mais qui finira par s'élargir en union de toute l'humanité.

Nous allons le voir, marqué par son enfance dans les ghettos, se passionner tour à tour ou même parfois simultanément pour : l'hébreu que peut-être dès 1874, soit à la même époque que le "rêve » de BenYehouda ${ }^{4}$, et donc avant le premier article de celui-ci (1879), le lycéen

\footnotetext{
* Chercheur indépendant, dernier rattachement : Paris 13 (2012).

${ }^{1}$ Je remercie Christer Kiselman, Roy McCoy, Ionel Oneţ, et Tsvi Sadan pour leur collaboration.

${ }^{2}$ Cf. esperanto-france.org/IMG/pdf/programo_unesko_zamenhof_2017.pdf (12 p.).

${ }^{3}$ L. L. Zamenhof, Le Hillélisme : Projet de solution $\overline{\text { de }}$ la question juive, trad., intr. et notes de Pierre Janton, Clermont-Ferrand, Ass. des Publications de la FLSH, coll. FLSH de l'Univ. Blaise-Pascal, NS 44, 1995, p. 87. Original: Гомо сумъ [= Homo sum, pseud. de L. Zamenhof], Гиллелизмъ [Gillelizm]: Проектъ решения еврейскаго вопроса, Варшава, Вейденфельдъ, [1901], 78 р., BN d'Israël, 23 В 13780.

${ }^{4}$ De 1875, selon son «Ha-Halom ve-Shivro » [Le songe et sa réalisation], Ha-Toren, déc. 1917: «J'ai fait un rêve. [...] depuis ce moment merveilleux de ma vie [...] seulement 43 ans ou presque se sont écoulés ». (Trad. Désirée Mayer, «Éliezer Ben
} 
Zamenhof aurait déjà « espéré faire revivre en tant que langue parlée » ${ }^{5}-$ le sionisme avant la lettre, après une phase pré-sioniste penchant pour les USA, l'espéranto et surtout son hillélisme ${ }^{6}$ dont nous étudierons le double texte fondateur et l'évolution universalisante.

C'est le cours de toute une vie que, dans sa relation avec le judaïsme, nous serons ainsi amenés à survoler puisqu'il a écrit, à un correspondant de Boulogne-sur-Mer :

Je suis juif et tous mes idéaux, leur naissance, maturation et opiniâtreté, toute l'histoire de mes luttes permanentes, au dedans et au dehors: tout est inséparablement lié à cette judéité qui est mienne [...]

Ma judéité a été la cause principale pour laquelle, depuis la plus tendre enfance, je me suis totalement consacré à une idée primordiale, un rêve unique : au rêve de l'union de l'humanité.

\section{Espoir de faire revivre l'hébreu comme langue parlée}

Dans une lettre de 1895 où il tait son identité juive, Zamenhof écrit :

Lorsque je suis passé [...] au $2^{\mathrm{e}}$ lycée classique de Varsovie, j'ai été quelque temps séduit par les langues antiques et j'ai rêvé un jour d'aller dans le monde entier et, par des discours enflammés, d'incliner les gens à faire revivre une de ces langues pour un usage commun. Ensuite, je ne me souviens déjà plus de quelle façon, j'en suis venu à la ferme conviction que c'est impossible. ${ }^{8}$

Yéhouda: Le combat pour la renaissance hébraïque », Mémoires de l'Académie nationale de Metz, Metz, 2008, p. 192).

En tant qu'historien, par fidélité aux sources nous reproduirons les transcriptions latines de l'hébreu ou du yiddish telles qu'elles figurent dans les textes consultés (exceptés ceux en espéranto).

5 «I did [...] hope to revive Hebrew as a spoken language among my coreligionists. » («Esperanto and Jewish Ideals: Interview for The Jewish Chronicle with Dr. Zamenhof», The Jewish Chronicle, 6-9-1907, p. 16, notre trad.).

${ }^{6}$ Éthique fondée sur l'enseignement de Hillel l'Ancien au $\mathrm{I}^{\mathrm{er}}$ siècle énoncée ainsi : « Ne fais pas à autrui ce que tu ne voudrais que l'on te fasse ».

7 Lettre à Alfred Michaux, 21-2-1905, dans Gaston Waringhien, Leteroj de L.-L. Zamenhof: La tragedio de lia vivo rivelita de lia jus retrovita korespondo kun la francaj eminentuloj [Lettres de L.-L. Zamenhof: La tragédie de sa vie révélée par sa correspondance retrouvée avec les sommités françaises], Paris, Sennacieca Asocio Tutmonda [Association mondiale à caractère anational], 1948, vol. I : 1901-1906, p. 106-107. (Notre trad.) Car sans doute peu de lecteurs la comprennent, en général nous ne reproduirons pas les originaux dans cette langue (traduits par nous le plus litt. possible). Dans les notes, « esp. » signifiera espéranto.

${ }^{8}$ Lettre à [N. A.] B[orovko], publiée en esp. dans Lingvo Internacia, I, n ${ }^{\circ}$ 6-7, juinjuillet 1896, p. 116, BN d'Autriche, 700038-B.1895-1898; notre trad. 
Plus tard, dans une interview en 1907 pour un hebdomadaire juif anglais, il nomme la langue et spécifie le champ d'action, les juifs du monde entier: " Il est certain que j'avais, à une époque, espéré faire revivre l'hébreu en tant que langue parlée chez mes coreligionnaires, mais j'ai bientôt été convaincu que c'était impossible ${ }^{9}$.

Zamenhof a passé l'examen d'entrée au lycée en août $1874^{10}$. Son rêve et le bref espoir, formulé dans la seconde citation, ne faisaient qu'un car le net parallélisme entre leur fin respective incline à penser que ces expressions quasi identiques s'appliquaient à une seule et même langue antique. Ils dateraient donc de la seconde moitié de 1874, alors que son père achevait la première partie d'une « Histoire biblique abrégée $»{ }^{11}$.

Norman Berdichevsky, ancien maître de conférences d'études juives à l'université de Floride centrale, écrit : «En diverses occasions Zamenhof aussi avait caressé l'idée de faire revivre l'hébreu ${ }^{12}$. La seconde occasion, vu la coïncidence, pourrait avoir été le premier article de Ben-Yehouda, de 1879, car Zamenhof a également indiqué que, « étudiant à l'Université [où il est entré début septembre 1879], bien que je fusse destiné à la profession médicale, j'avais consacré une attention considérable aux langues: latin, grec, français, allemand, anglais et hébreu $)^{13}$.

Et voici comment le jeune Zamenhof en était venu à l'idée de renouveau de l'hébreu dont l'abandon fut, selon lui, l'expérience la plus douloureuse de l'exil, préfigurée par le récit de la Tour de Babel :

En tant que juif [...], nous avons ressenti plus que quiconque toute la malédiction de la multiplicité des langues humaines et, de tous les malheurs juifs, le plus grand, qui toujours se montrait à nous, c'est que les juifs sont sans

\footnotetext{
9 « I did certainly, at one time, hope to revive Hebrew as a spoken language among my coreligionists, but I soon became convinced that that was impossible ». («Esperanto and Jewish Ideals », op. cit., p. 16, notre trad.).

${ }^{10}$ D-ro Leono [Lev] Zamenhof, «El la Biografio de D-ro L. L. Zamenhof», Universo, vol. II, 1910/1911, p. 170.

${ }^{11}$ Сокращенная библейская история [Sokraščennaja biblejskaja istorija], Варшава [Varšava], 1875, $52 \mathrm{p} ., 11^{\mathrm{e}}$ édition posthume en 1908.

12 «At various times Zamenhof, too, had toyed with the idea of reviving Hebrew ». Norman Berdichevsky, «Esperanto and Modern Hebrew : 'Artificial' Languages that Came to Life », New English Review, fév. 2014, newenglishreview.org/custpage.cfm/frm/160333 consulté le 19-2-18; notre trad.

${ }^{13}$ « As a student at the University, although I was intended for the medical profession, I had devoted considerable attention to languages: Latin, Greek, French, German, English and Hebrew ». « Esperanto and Jewish Ideals », op. cit., p. 16.
} 
langue. Nous avons toujours senti que la langue est le bien le plus sacré de l'homme et que les juifs, justement, en sont privés. Nous n'avons envié ni le pouvoir politique des peuples ni leur richesse-nous avons envié la santé spirituelle de leur "moi », leur langue, leur littérature. Il nous a été pénible de constater combien les juifs écorchent diverses langues sans en posséder bien une seule ni avoir la possibilité de se donner entièrement à aucune, car chacune d'elles est l'incarnation de la vie, de l'esprit, de l'histoire spécifique d'un peuple et, par là, radicalement étrangère aux juifs. À la lumière de ces réflexions et d'autres semblables, nous avons envisagé quelque temps de faire revivre l'hébreu ancien. $^{14}$

Les raisons qui lui ont fait abandonner cette idée, bien que l'histoire lui ait par la suite donné tort, étaient que « l'hébreu ancien, langue morte qui ne nous est pas parvenue dans la plénitude de son expressivité [...] ne convient absolument pas pour le rôle de langue maternelle vivante ${ }^{15}$; car, selon lui qui pour sa part en avait « une connaissance intime ${ }^{16}{ }^{-}$il réalisera une traduction de la Bible hébraïque en espéranto ${ }^{17}$, qui lui vaudra cet éloge : "La traduction espéranto de Zamenhof [pourtant sans notes] est le commentaire de l'Ancien Testament le plus bref et le meilleur qui me soit jamais venu entre les mains $»^{18}-$, « l'acquisition des rudiments de cette langue est d'une difficulté si extraordinaire », qu'elle n'est accessible « qu'à une toute petite poignée de spécialistes passionnés mais nullement aux masses dans leur ensemble $»^{19}$.

Lui-même, par exemple à l'occasion des deux derniers congrès universels d'espéranto auxquels il a pu participer, en août 1912 et 1913, a été appelé à la Torah à la synagogue de Cracovie ${ }^{20}$ puis, l'année suivante,

\footnotetext{
${ }^{14}$ L. L. Zamenhof, Le Hillélisme, op. cit., p. 107. (Trad. modifiée par nous grâce à la nouvelle trad. esp. d'A. Korženkov dans L.-L. Zamenhof, Mi estas homo: Originalaj verkoj [Je suis un homme: Euvres originales], Kaliningrado, Sezonoj, coll. Scio 6, 2006, p. 82-83).

${ }^{15}$ Ibid., p. 105.

16 "The Hebrew, with which he has an intimate acquaintance». "The Bible in Esperanto », The Jewish chronicle, 30-8-1907, p. 18, notre trad.

${ }^{17}$ Les premiers livres en ont été publiés chez Hachette et l'ensemble par la British and Foreign Bible Society.

18 "Zamenhof's Esperanto translation is the briefest and best commentary on the Old Testament which has ever come into my hands». Rev. J. Beveridge, B.D., A.E., [examinateur d'hébreu dans des univ. britanniques], dans Joseph Silbernik, "Zamenhof the idealist », Amerika Esperantisto / American Esperantist, vol. 22, n 3, avril 1918, p. 9-10. (Les italiques correspondent à des caractères gras dans l'article. Notre trad.).

${ }^{19}$ L. L. Zamenhof, Le Hillélisme, op. cit., p. 106.

${ }^{20}$ Lettre de [Maurice ?] Salzmann à Roland Jossinet, du 5-10-1952; citée par Waringhien dans N. Z. Maimon, La Kasitia vivo [vie cachée] de Zamenhof, Tokyo: Japana Esperanto-Instituto, 1978, p. 187.
} 
à celle de Berne où il a «fait entendre les bénédictions du 'Maftir' ${ }^{21}$. Et, au moins à la fin de sa vie, il a bien, ne serait-ce qu'une fois, parlé hébreu, en décembre 1916 chez le Dr Volf à Białystok : " Soudain le Dr Zamenhof s'est tourné vers moi et m'a demandé quelle langue je parlais. Quand je lui ai répondu que j'apprenais l'hébreu, il s'est aussitôt mis à parler hébreu avec moi $»^{22}$.

Zamenhof est donc l'un des premiers locuteurs de l'hébreu, encore très peu nombreux en Russie car la plupart se trouvait surtout en Palestine $^{23}$. Et d'autres espérantistes en ont été des promoteurs actifs, dont Ben-David (l'enseignant Lev Davidovitch), qui « a fondé le premier club en Russie de conversation dans cette langue antique $»^{24}$, sans doute à Odessa, et en 1897 au plus tard puisqu'il y est mort le 20 décembre de cette année-là.

\section{Pré-sionisme ou « territorialisme » avant la lettre}

Zamenhof avait aussi «travaillé pendant trois ans sur le yiddish ${ }^{25}$, à une époque où,

...lorsqu'il était étudiant, [...] il croyait qu'il ne pourrait rien y avoir de bon pour les juifs jusqu'à ce qu'ils aient leur propre terre, une colonie ou quelque chose de similaire. Et pendant un certain temps il avait beaucoup étudié la question et s'était occupé de colonisation agricole par des juifs dans le sud de la Russie (Ukraine). ${ }^{26}$

\footnotetext{
${ }^{21}$ Cité dans Adolf Holzhaus, « Domoj, urboj, en kiuj Zamenhof loĝis, studis », Israela Esperantisto, $\mathrm{n}^{\circ}$ 59, déc. 1976, p. 13, notre trad.

22 Joel Kavenaki, « Neforgesebla renkontiĝo kun d-ro Zamenhof », Israela Esperantisto, $\mathrm{n}^{\circ}$ 59, déc. 1976, p. 2, notre trad.

${ }^{23}$ Roberto Bachi, 1974, World Population Year : The population of Israel [Année mondiale de la population : La population d'Israël], [Jerusalem], C.I.C.R.E.D. [Comité Int. de Coop. dans les REcherches nationales en Démographie] Series, 1977, (nos trad.) : il y avait alors en Palestine « environ 34000 [...] juifs parlant hébreu comme langue unique ou maternelle », p. 287 : about 34,000 [...] Jews speaking Hebrew as only or first language), "ou quelque $0,25 \%$ des juifs du monde en ce temps-là », p. 286 : or some $0.25 \%$ of the World Jewery at that time.

${ }^{24}$ N. Slouŝĉ (Nahum Slousch, qui allait être professeur de la chaire de la langue hébraïque à la Sorbonne (1904-1919), «Lev Davidoviĉ $\uparrow$ », Lingvo Internacia, n²7, mars 1898 , p. 33 ;

http://anno.onb.ac.at/cgi-content/anno-plus?aid=e0b\&datum=1898\&page $=44$ (19-7-2018).

${ }^{25}$ « for three years, I worked with Yiddish », « Esperanto and Jewish Ideals », op. cit., p. 16 , notre trad.

${ }^{26}$ E[dward] Wiesenfeld, «La Galerio de Zamenhofoj: D-ro Ludoviko Zamenhof», Heroldo de Esperanto, $\mathrm{n}^{\circ}$ 231, 10-1-1925, p. 1.
} 
Mais vers le printemps 1881, il a organisé une réunion de quinze étudiants :

...et je leur ai dévoilé un plan que j'avais conçu, de fonder une colonie juive sur quelque portion inoccupée du globe, qui serait le commencement, et deviendrait le centre, d'un État Juif indépendant [...] et nous avons formé ce qui, je crois, a été la première organisation politico-juive en Russie. ${ }^{27}$

Cela s'est passé «quelques mois » ${ }^{28}$ avant qu'il ne quitte Moscou (ce qu'il a fait entre le 22 mai/3 juin et le 22 juillet/3 août 1881$)^{29}$ donc, au plus tard, juste après le début, le 15/27 avril, des pogroms ayant suivi l'assassinat d'Alexandre II. Et si la colonie alors envisagée ne se localise déjà plus en Ukraine, c'est sans doute que la série de pogroms, ayant bientôt fait tache d'huile à partir d'Elisabethgrad, venait de mettre fin à ce projet-là. Son éphémère organisation est donc née près d'un an et demi avant la publication, à Berlin en septembre 1882, d'Autoémancipation du Dr Leo Pinsker d'Odessa. Mais, en 1881, une autre association a été créée à Saint-Pétersbourg, elle aussi par des étudiants ${ }^{30}$, après les pogroms ${ }^{31}$ : Ahavat Zion [Amour de Sion]. Une chronologie fine resterait donc à établir sur ce point.

\footnotetext{
${ }^{27}$ « I convened a meeting of fifteen of my fellow-students, and unfolded to them a plan which I had conceived of founding a Jewish colony in some unoccupied portion of the globe which would be the commencement, and become the centre, of an independent Jewish State [...] and we formed what I believe was the first politico-Jewish organisation in Russia ». «Esperanto and Jewish Ideals », op. cit., p. 17, notre trad.

28 《 a few months » Ibid.

${ }^{29}$ Un « Billet (Билеть) de l'Université Impériale de Moscou », l'autorisait à se rendre à Varsovie entre la date de la signature du billet, le 22-5[/3-6]-1881, et le 15[/29] août. Et le 22-7[/3-8], c'est déjà sur place qu'il signe une demande au recteur de celle de Varsovie, d'être compté au nombre des étudiants de $3^{\mathrm{e}}$ année. (Juntaro Iŭaŝita (eld.), Halina-Dokumento pri la studentaj jaroj de L. L. Zamenhof: Oficialaj arkivoj gimnaziaj kaj universitataj en la rusa lingvo. La originaloj esploritaj kaj tajpitaj de Halina [Edelbaum], la Esperanto-tradukoj ankaŭ faritaj de Halina, la komentaro verkita de Naokazu Kawasaki, Tokio, Vendejo (lieu de vente). [Document sur les années étudiantes de L. L. Zamenhof: Archives officielles du lycée et des universités, en russe avec trad. esp. par Halina], Osaka, Esperanta Servo-Centro, 1977, p. 176-177 et 172-173).

${ }^{30}$ Élie Barnavi, Israël au XXe siècle, Paris, PUF, coll. L'Historien, 1982 (1 $1^{\text {ère }}$ éd.), éd. numérisée FeniXX, $5^{\mathrm{e}}$ p. du chapitre I, 3 : « Le choix sioniste ».

31 «Tiomkin, Vladimir», à jewishvirtuallibrary.org/tiomkin-vladimir (22-7-18); confirmé en croisant avec «[Temkin, Vladimir Ionovitch]», dans Еврейская энциклопедия [ЕЭБЕ : Encyclopédie juive, Saint-Pétersbourg, Brockhaus et Efron, t. XIV, 1913, col. 798].
} 
Parmi d'autres raisons visant à expliquer pourquoi le jeune Zamenhof a demandé, en cours d'études, à changer d'université, la dernière en date est que son activisme à Moscou l'avait distrait de ses études et sans doute même mis en danger ${ }^{32}$. Ajoutons une fâcheuse coïncidence. L'assassin d'Alexandre II avait achevé ses études secondaires à Biélostok en 1875 dans l'établissement même (l'actuel Lycée $n^{\circ} 6$ Sigismond II Auguste $)^{33}$ qu'avait fréquenté jusqu'à la fin de l'année $1873^{34}$ Lejzer (comme se prénommait alors Lazar Zamenhof). Ils ne s'étaient sans doute pas connus puisque Lejzer se trouvait probablement trois classes en dessous de lui, mais l'éventuel rapprochement aurait pu lui être fatal vu l'antisémitisme le moins raisonné et le plus débridé qui faisait alors rage.

Lazar, rentré chez ses parents, semble ne plus avoir fait parler de lui pendant six mois, jusqu'au pogrom de Noël 1881 à Varsovie même ${ }^{35}$. Il a alors écrit un long article publié dans quatre numéros successifs de Rassvet, "Que devons-nous donc finalement faire ?» ${ }^{36}$. Il y appelait à choisir un endroit à faible densité humaine aux USA, pour en faire un territoire à grande majorité juif puis, une fois atteint le nombre des 60.000 habitants nécessaires, demander que, selon la Constitution, il soit reconnu comme nouvel État, juif, de $1^{\prime} U_{n i o n}^{37}$ (comme les mormons étaient en passe de l'obtenir pour leur territoire de l'Utah colonisé depuis 1847).

\footnotetext{
32 «More likely, his activism had left him distracted, exposed, and endangered ». Esther Schor, Bridge of Words : Esperanto and the Dream of a Universal Language [Pont de mots : L'espéranto et le rêve d'une langue universelle], New York, Metropolitan Books, 2016, p. 66.

33 «Junior High School No 6 (the former Białystok Junior High School which the creator of Esperanto Ludwik Zamenhof and Ignacy Hryniewiecki had attended)». «Białystok: School of Dialogue at Stanisław Staszic Vocational-Technical and High School Complex with Special Educations Sections », 2013; dialog.org.pl/szkoladialogu/en/szkola/bialystok-2/.

${ }^{34}$ Lettre à A. Michaux, 21-2-1905, dans G. Waringhien, Leteroj de L.-L. Zamenhof, op. cit., p. 111-112, notre trad.

${ }^{35}$ «Les scélérats ont voulu pénétrer dans le quartier de Nalewki, cœur de la vie juive à Varsovie, où habitaient aussi les Zamenhof, mais de solides portefaits juifs se sont opposés à eux et les ont repoussés ». N. Z. Maimon, La Kaŝita, op. cit., p. 88, notre trad. Pour les détails, cf. « Pogrom à Varsovie », Rassvet, $n^{\circ}$ 51, 18[/30]-12-1881. Trad. esp. dans Adolf Holzhaus, Doktoro kaj lingvo [Docteur et langue] Esperanto, Helsinki, Fondumo Esperanto, 1969, p. 121.

36 Гамзефон [Gamzefon/[H]amzefon, anagramme et pseud. de Заменгоф: Zamengof/Zamenhof], «Что же наконец делать ? " [Chto že nakonets delatj ?], Paccвem [Rassvet], $\mathrm{n}^{\circ} 2$ à 5 de 1882, du 12/24-1 au 1/13-2- 1882 ; retrad. en esp. sous le titre « Kion do finfine fari ? » dans L.-L. Zamenhof, Mi estas homo, op. cit., p. 5-22.

${ }^{37}$ Ibid., p. 19.
} 
Implicitement il s'y disait prêt à partir aux USA car il le terminait par une envolée s'opposant au traditionnel «L'an prochain à Jérusalem ! ». Envolée d'autant plus provocante qu'elle faisait clairement allusion à d'autres fleuves, ceux de Babylone au bord desquels « nous étions assis et pleurions, nous souvenant de Sion $»^{38}$, et supposait donc qu'au contraire là-bas tous pourraient enfin oublier Sion, puisque $\mathrm{y}$ « commen[çant] une nouvelle vie, glorieuse $»{ }^{39}$ dans les chants de joie car sans plus d'oppresseurs : «Au revoir, mon peuple, dans la propre chaumière sur les libres rives du Mississipi ! ${ }^{40}$.

\section{Sionisme avant la lettre, ou : « proto-sionisme »}

Ayant été critiqué, entre autres par M. L. Lilienblum ${ }^{41}$ qui allait devenir secrétaire des « Amants de Sion », Zamenhof s'est rallié alors au choix de la Palestine. Le titre de son nouvel article, "Sous l'étendard commun » ${ }^{42}$, exprimait bien son désir d'unité, malgré ces « inconvénients qui nous attendent en Palestine en raison des sanctuaires d'autres peuples, et d'espoirs et aspirations difficilement réalisables que quelques partis hébreux apporteront avec eux sur la terre de nos ancêtres ${ }^{43}$. Ces « espoirs et aspirations » paraissent renvoyer à un passage de son article précédent : «Je ne parlerai pas [...] des zélotes de l'hébreu ancien [...] Rappelons seulement, en passant, qu'il y a un parti de gens qui croient la restauration de la [patrie en] Palestine inimaginable et absurde sans la reconstruction du temple et de l'antique culte mosaïque ${ }^{44}$.

Mais la principale raison de ce brusque retournement aura été la venue d'étudiants délégués de BILU ${ }^{45}$, tout juste fondé à Kharkov le 21 janvier $1882^{46}$, qui l'avaient soudain sorti de son isolement, d'autant

\footnotetext{
${ }^{38}$ Psaume 137, 1.

${ }^{39}$ Gamzefon, « Kion do », op. cit., p. 22, notre trad.

${ }^{40}$ Ibid., notre trad.

${ }^{41}$ N. Z. Maimon, La Kaŝita, op. cit., p. 93-95.

${ }^{42}$ Гамзефон [L. Zamenhof], « Под общее знамя! » [Pod obščee znamja], Rassvet, $\mathrm{n}^{\circ} 13,26-3[/ 7-4]-1882$; retrad. en esp. sous le titre « Sub la komuna standardo ! » dans L.-L. Zamenhof, Mi estas homo, op. cit., p. 22-26. Notre trad.

${ }^{43}$ Ibid., notre trad.

${ }^{44}$ Gamzefon, « Kion do », op. cit., p. 18, notre trad.

${ }^{45}$ Association de sionistes, dont le nom est un acronyme des premiers mots d'Isaïe 2,5,

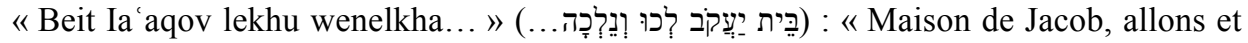
marchons... ». Cf. Jean-Marie Delmaire (présentés par), "Statuts et règlement interne des Biluim (1883)», Tsafon, n $^{\circ} 13$, automne 1993, p. 109-122.

46 [Getzel Kressel], "Bilu », Encyclopedia Judaica, The Gale Group, 2008 ; jewishvirtuallibrary.org/bilu-2 (14-2-18).
} 
plus grand que le petit groupe, d'étudiants aussi, qu'il avait constitué à Moscou ne semble pas avoir survécu à son départ un peu précipité ; d'où son soudain lyrisme, et le nouveau fleuve évoqué :

Ce qui, il y a peu, semblait presque un rêve irréalisable, a maintenant lieu sous nos yeux: l'allant pour la Palestine commence à pénétrer dans la masse populaire, des gens s'élancent vers la Palestine non seulement en rêve, mais ils ont le courage de planter leurs tentes sur les bords du Jourdain. ${ }^{47}$

Voici, en effet, ce qu'il répondit, le 18[/30] novembre 1883, aux membres de BILU déjà au Proche-Orient :

La réception de votre lettre m'a fait très plaisir : j'ai alors ressenti ce que ressent un exilé en recevant une lettre de sa lointaine patrie [...] Il y a deux ans environ, quand vos délégués étaient à Varsovie, racontant l'activité de votre petit cercle et recrutant des volontaires, je me suis, malgré le scepticisme que m'avait donné ma pesante expérience, tellement enthousiasmé que dans [l'impulsion du] premier moment j'avais presque décidé de dire adieu à mon passé, de négliger les longues années durant lesquelles je m'étais préparé pour un champ d'activité défini, et de partir - seulement pour commencer à travailler en Palestine deux ans plus tôt. ${ }^{48}$

Il était alors en troisième année de médecine (dont les études duraient cinq ans). Et il venait d'adhérer à Hibbat Zion [Amour de Sion $]^{49}$, dont il avait fondé début mars, avec des étudiants, la première section à Varsovie, Še 'erit yiśra'el [reste d'Israël] ${ }^{50}$, justement pour soutenir les émigrants de BILU - groupe de jeunes célibataires socialistes de Hibbat Zion (de Kharkov puis aussi de Moscou) - , par des cotisations individuelles mensuelles, transmises illégalement par Zamenhof au rabbin Adolf Salvendi de Bad Dürkheim servant d'intermédiaire. Hebdomadaires et secrètes, les réunions de l'association se tenaient à

\footnotetext{
${ }^{47}$ Gamzefon, « Sub la komuna standardo ! », op. cit., p. 26, notre trad.

${ }^{48}$ L. M. Zamenhof, lettre du 18[/30]-11-1883 aux membres de BILU [en Eretz Israel], conservée au Centre Goldstein-Goren de recherche sur la Diaspora de l'Univ. de TelAviv, catalogué à «P2:The Shlomo Zalman Zuckerman Papers (archive materials) » $\left(n^{\circ} 000240751\right)$, p. 40 , date «30.11.1883», n 372

(alephprd.tau.ac.il/exlibris/aleph/a22_1/apache_media/BMVEC9S9I15X2DFL6NII6CH 1DC9BV6.pdf). Trad. esp. du ms. original dans L.-L. Zamenhof, Mi estas homo, op. cit., p. 27, notre trad.

${ }^{49}$ Sur ce mouvement, voir, sous la direction de Roland Goetschel, la thèse de JeanMarie Delmaire, De Hibbat-Zion au sionisme politique, soutenue à Strasbourg II en 1986, Lille, A.N.R.T., 1990.

${ }^{50}$ Citation de Michée 2, 12 : «J'aurai soin de te rassembler tout entier, ô Jacob, de te réunir, ô reste d'Israël ! ». Trad. du rabbinat.
} 
chaque fois chez une personne différente ${ }^{51}$. Et un réseau de filiales s'était bientôt étendu dans « de nombreuses villes de Pologne et de l'Ouest de la Russie » ${ }^{52}$. Mais, au moins à Varsovie : "Avec une immense tristesse on doit noter ce fait navrant, et même vraiment incroyable, que et les hassidim polonais et ceux qu'on appelle les Allemands juifs [sic, et non : juifs allemands] ne prennent presque aucune part aux affaires juives et restent totalement indifférents à notre égard ${ }^{53}$.

La division était renforcée par la diversité de langues, ce qui explique qu'une réunion y soit restée sans résultat immédiat malgré la présence de celui qui était à la tête du mouvement Hibbat Zion dans toute la Russie, venu spécialement pour l'occasion :

Zamenhof vint aussi, accompagné de quelques étudiants [litvaks], en tenue d'étudiant, et s'assit à la table de présidence. Les assistants pieux [comprendre : hassidim polonais] ne les reçurent pas favorablement. Mais le rabbin Mohilewer [de Biélostok, donc litvak comme eux] les accueillit très gentiment, avec amour et bienveillance. $^{54}$

Et, le mois suivant, ont finalement été fondées non pas une, mais deux autres sociétés sionistes (en plus de celle des étudiants), l'une de hassidim polonais, et l'autre de mitnagdim [《opposants » aux premiers] principalement litvaks comme Zamenhof, et qui deviendra la plus importante. En grande partie grâce à lui celle-ci se fondra finalement avec son Š 'erit yiśra'el pour donner naissance, le 18 août 1883, à l'association unitaire des Hovevei Zion [Amants de Sion] de Varsovie, dont il se retrouve aussitôt «à la tête de l'exécutif » ${ }^{55}$ et qui, de 160 membres à sa fondation, serait passée à 400 en trois mois $^{56}$.

Mais fin novembre 1883, bien qu'il écrive encore «comme votre ami sincère, comme votre frère dans les idées et aspirations, peut-être

51 Voir N. Z. Maimon, La Kaŝita, op. cit., p. 100-101, et Aleksander Korženkov, Homarano : La vivo, verkoj kaj ideoj de d-ro [Homarane : Vie, œuvres et idées du $\mathrm{D}^{\mathrm{r}}$ ] L. L Zamenhof, Kaliningrad / Kaunas: Sezonoj / Litova Esperanto-Asocio, coll. Scio 8, 2009, p. 49-50.

52 «Esperanto and Jewish Ideals », op. cit., p. 17, notre trad.

53 Nahum Iš-Gamzu [qui pourrait être un pseud. de Zamenhof], dans Nedeljnaja Khronika Voskhoda, $\mathrm{n}^{\circ} 17,14[/ 26]-4-1882$, col.452; cité en trad. esp. dans A. Korženkov, Homarano, op. cit., p. 286, notre trad.

${ }_{54}^{5}$ N. Z. Maimon, La Kaŝita, op. cit., p. 101, notre trad.

55 « at the head of the Executive ». « Esperanto and Jewish Ideals », op. cit., p. 17, notre trad.

${ }^{56}$ Selon N. Z. Maimon, La Kasitita, op. cit., p. 102. 
comme votre futur compagnon ${ }^{57}$ aux membres de BILU au ProcheOrient (la première quinzaine d'entre eux ayant débarqué à Jaffa le 6 juillet de l'année précédente $)^{58}$, le dernier paragraphe, conclusion de sa longue lettre, commence par: «J'attends impatiemment les statuts et le programme, que vous aviez promis, de votre action future. Je serais très heureux si vous me donniez quelque possibilité de rétablir votre crédit ici. Jusqu'à maintenant nous ne savons rien de concret sur vous ${ }^{59}$, à part le fait que dans la fougue de leur jeunesse et en raison de leur «manque d'expérience » ils refusent tout compromis, « dans votre dernière lettre il est écrit que Rothschild veut loger 30-50 membres de Bilu à Rishon-leZion $^{60}$, mais vous avez refusé ! Qu'avez-vous fait? Enfin vous aviez la possibilité de vous établir sur un terrain ferme, mais vous avez refusé ${ }^{61}$; cela alors que diminuaient de manière préoccupante les sommes qu'il s'efforçait de lever pour eux : «Maintenant les rentrées sont d'environ 250-300 roubles par mois $»{ }^{62}$.

Six mois plus tard, à la fin de l'année 1883-1884, Lazar achevait ses études de médecine ${ }^{63}$. Mais il n'est pas parti retrouver ses amis et frères de BILU qui pourtant, «se joignirent à la colonie de Gederah (qui avait été lancée par l'association des Hovevei Zion de Paris) » ${ }^{64}$, après un voyage organisé avec Ben-Yehouda, et parlant hébreu entre eux ${ }^{65}$; ce qui pourrait avoir été pour Lazar une troisième occasion de se mettre à la pratique de l'hébreu parlé, tant qu'il n'avait pas renoncé à devenir leur compagnon en Palestine.

\footnotetext{
${ }^{57}$ L. M. Zamenhof, lettre du 18-11-1883, traduction espéranto du manuscrit original dans L.-L. Zamenhof, Mi estas homo, op. cit., p. 30, notre trad. de l'esp.

${ }^{58}$ G. Kressel, « Bilu », loc. cit.

${ }^{59}$ L. M. Zamenhof, lettre du 18-11-1883, traduction espéranto du manuscrit original dans L.-L. Zamenhof, Mi estas homo, op. cit., p. 31. Notre trad. Ironie du destin, dans le même document où est cataloguée cette lettre (voir note 48), sont catalogués, p. 1-2, sous les $\mathrm{n}^{\circ} 14-15$, les « Statuts pour la société 'Viens maison Israel'[sic]» (dont BILU est l'acronyme), et leur version allemande ( $\mathrm{n}^{\circ} 10$ et 11$)$.

${ }^{60}$ [Première à Sion], devenue une ville de $250.000 \mathrm{~h}$.

${ }^{61}$ L. M. Zamenhof, lettre du 18-11-1883, traduction espéranto du manuscrit original dans L.-L. Zamenhof, Mi estas homo, op. cit., p. 29, notre trad.

${ }^{62}$ Ibid., p. 30, notre trad.

${ }^{63}$ Derniers examens connus : le 14[/26]-6-1884 ; grade de médecin obtenu le 5[/17]-285 selon son diplôme du 28-2[/12-3]. J. Iŭaŝita, Halina, op. cit., p. 220-223 et 234-237.

64 «which was started by the Chovevei Zion Society of Paris ». Herman Rosenthal, "Agricultural colonies in Palestine», dans The unedited full-text of the 1906 Jewish Encyclopedia [Le texte complet inédit de l'Encyclopédie juive de 1906] (jewishencyclopedia.com/articles/907-agricultural-colonies-in-palestine).

${ }^{65}$ G. Kressel, « Bilu », loc. cit.
} 
En février 1885 il s'était installé comme médecin de campagne à Veisiejai, ce qui l'éloignait à la fois physiquement (il s'agissait d'une petite bourgade lituanienne) et mentalement des Hovevei Zion de Varsovie, avec lesquels il restait sporadiquement en contact. Et fin 1886, ouvrant à Varsovie un cabinet d'oculiste, c'est chez l'un d'eux, Josef Levite, qu'il dit avoir fait la connaissance de sa future épouse ${ }^{66}$. Mais, comme il l'écrira à Michaux :

...après 3-4 ans de travail énergique pour le sionisme j'en suis arrivé à la conviction que cette idée ne mènera à rien, et c'est pourquoi j'ai rejeté cette idée, bien que dans mon cœur elle me soit toujours restée chère, comme un rêve agréable mais irréalisable; lorsqu'en 1897 est né le grand mouvement sioniste organisé par Herzl, je ne pouvais déjà plus y adhérer. ${ }^{67}$

L'année suivant le premier congrès sioniste (Bâle, 1897), à « la première conférence des Sionistes Russes à Varsovie ${ }^{68}$ (une semaine avant le second Congrès de Bâle), Zamenhof a commencé son «brillant discours, qui l'a rendu célèbre dans les sphères concernées ${ }^{69}$, en déclarant :

Frères juifs ! [...] Nous avons renoncé à cette idée [le sionisme], non pour un amour insuffisant envers elle, mais seulement en raison de preuves rationnelles impossibles à éconduire et qui nous ont définitivement convaincu que le sionisme n'est que le fruit tentant d'une insuffisante compréhension de l'essence du problème juif. ${ }^{70}$

\footnotetext{
${ }^{66}$ Lettre à A. Michaux, 21-2-1905, dans G. Waringhien, Leteroj de L.-L. Zamenhof, op. cit., p. 112, notre trad.

${ }^{67}$ Ibid., p. 109, notre trad.

${ }^{68}$ « the first Conference of Russian Zionists in Warsaw in 1898 », Stanley Nash, « Ahad Ha-Am and 'Ahad Ha-Amism' ", dans Jacques Kornberg (ed.), At the Crossroads : Essays on [Au carrefour : Essais sur] Ahad Ha'am, Suny Press, 2012, p. 74 ; (du 7/19 au 10/22-8-1898), Michael Heymann, The Uganda Controversy [La polémique sur l'Ouganda], vol. 1, Transaction Publishers, 1970, p. 5.

${ }^{69}$ E. Wiesenfeld, «La Galerio », op. cit., p. 1, qui nomme la rencontre : «Premier congrès sioniste à Varsovie ».

70 «Alvoko al la juda intelektularo», trad. esp. par Adolf Holzhaus, dans $\hat{G}$ is la homaranismo [Jusqu'à l'homaranisme] : 1896-1906, Tokyo, Eldonejo Ludovikito, coll. Iom reviziita plena verkaro de L. L. Zamenhof [par la suite abrégée en : « coll. PVZ »], Originalaro 2, 1990, p. 1074-1075. Le professeur Janton a déjà montré que le ms. publié en russe et trad. en esp. par A. Holzaus est antérieur à la brochure Gillelizm de 1901 (cf. note 3), et il a inclus dans sa trad. fr. de cette brochure la trad. du ms. « qui en constitue le canevas et la trame, mais sur lequel nous possédons peu d'informations ». «Introd. » à L. L. Zamenhof, Le Hillélisme, op. cit., p. 17-18. L'information sur la conférence sioniste (contexte qui en éclaire l'entrée en matière) lui aura échappé. Ce ms.
} 


\section{Espéranto}

Entre-temps, avant même d'être marié, Zamenhof avait, en juillet 1887, « franchi le Rubicon » ${ }^{71}$ en publiant à compte d'auteur (grâce à une avance sur la dot) la première brochure d'espéranto, quasi simultanément en quatre langues : russe, puis polonais, français et allemand (et l'année suivante en hébreu et en yiddish, etc.) Certes, comme l'écrivait en 1907 le rabbin Harris, presque certainement devenu lui-même espérantiste :

...pour la communauté juive, les progrès de la nouvelle langue du Dr Zamenhof possèderont toujours un intérêt fascinant dans la mesure où l'espéranto doit son existence à ses efforts au bénéfice de la race juive. C'était la protestation d'un Juif au cœur généreux contre les débordements d'animosité dont son peuple a été la principale victime. L'invention d'un moyen de communication internationale était destinée à abattre la barrière des préjugés et des malentendus qui séparent les unes des autres les familles de la race humaine. C'était l'un des nombreux mouvements dont les Juifs de divers pays et diverses époques ont pris l'initiative pour le bien de l'humanité, et par lesquels ils ont réalisé l'ancienne prophétie de devenir « une Bénédiction pour d'autres ». ${ }^{72}$

Mais ce n'est pas ici le propos de traiter de sa diffusion, à laquelle a pourtant œuvré un bon nombre de juifs ${ }^{73}$ qui, amoureux du russe comme Zamenhof, ou « du polonais mais mal tolérés par les Polonais $»^{74}$ et/ou

autographe de 47 p. en russe est conservé à la BN d'Israël, Jérusalem, Archives Department, Abraham Schwadron Collection, [cote] Schwad 0107105.

${ }^{71}$ Lettre à B[orovko], dans G. Waringhien, Leteroj de L.-L. Zamenhof, op. cit., p. 350.

72 «For the Jewish community, the progress of Dr. Zamenhof's new language will always possess a fascinating interest inasmuch as Esperanto owes its existence to his strivings for the benefit of the Jewish race. It was the protest of the warm-hearted Jew against the racial animosities from which his people have been the principal sufferers. The invention of a medium of international communication was intended to break down the barriers of prejudice and misunderstanding that separate the families of the human race from one another. It was one of the many movements which the Jews of various countries and ages have led for the benefit of mankind, and by which they have realised the ancient prophecy of becoming 'a Blessing to others' ». "Esperanto and Jewish Ideals », op. cit., p. 16, notre trad.

${ }^{73}$ Principalement dans l'entre-deux-guerres (N. Berdichevsky, « Esperanto and Modern Hebrew », loc. cit. : « Jews constituted a large proportion of the pre-World War II Esperantists in Central and Eastern Europe.»). En 1907, l'estimation absolue était encore très modeste : « Je dirais qu'il y a au moins trois cents espérantistes juifs » : «I should say there are 300 or more Jewish Esperantists ». (L. Zamenhof , « Esperanto and Jewish Ideals », op. cit., p. 16, notre trad.).

${ }^{74}$ Jean-Claude Lescure, « L'espéranto, ou comment se protéger de l'exil (1900-1930)», Matériaux pour l'histoire de notre temps, «Pour une histoire de l'Exil français et belge », $\mathrm{n}^{\circ} 67,2002$, p. 21. 
les Russes, « effectuaient souvent un transfert linguistique en apprenant l'espéranto $\gg{ }^{75}$ et en participant activement à sa propagation. Nous ne donnerons qu'un extrait de l'interview de 1907 (où se trouve insérée sans guillemets la citation d'une lettre de Zamenhof de 1904 au rabbin Harris $^{76}$, que nous mettons en italiques) :

À l'origine c'était dans l'intérêt de mes coreligionnaires que j'ai inventé cette langue. Je les voyais coupés du reste du monde par une langue qu'ils ne parlaient qu'entre eux $[\ldots]$ Ce dialecte non seulement servait à les aliéner du monde des gentils mais même de leurs propres coreligionnaires dans d'autres pays, qui parlent la langue de ces pays et ne comprennent pas le yiddish. Ainsi le juif est séparé de son frère juif aussi bien que de son voisin chrétien. [...] Chaque fois que ma tâche me semblait désespérée, je n'avais qu'à penser à mes coreligionnaires, sans langue, et donc sans espoir de culture, dispersés dans le monde et pour cela incapables de se comprendre les uns les autres, et qui sont forcés de tirer leur culture de sources étrangères et hostiles, et la pensée de tout cela me remplissait d'une énergie renouvelée. Oui, je suis convaincu qu'il n'y a pas de peuple au monde pour lequel un moyen de communication mondial comme l'espéranto pourrait se montrer aussi utile que pour le peuple juif. Il permettrait de franchir l'abîme actuellement béant dans toutes les communautés juives entre le natif et l'étranger [il en avait, nous l'avons vu, lui-même fait l'expérience à Varsovie], le riche et le pauvre, le cultivé et l'inculte. ${ }^{77}$

\section{Hillélisme}

Cette nouvelle éthique, à l'origine en fait immémoriale, Zamenhof va indiquer la raison pour lui de l'appeler ainsi tout en énonçant le grand

75 Henri Minczeles, Vilna, Wilno, Vilnius, la Jérusalem de Lituanie, Paris, La Découverte, 1993, p. 354, cité par Jean-Claude Lescure, «L'espéranto, ou comment se protéger de l'exil (1900-1930)», op. cit, p. 21.

${ }^{76}$ I. Harris, "'Esperanto' and its Originator: How a Jew came to create a 'Universal Language' ", New Era Illustrated Magazine, vol. 6, n 2, janv. 1905, p. 142.

${ }^{77}$ " Primarily it was in the interests of my coreligionists that I invented this language. I saw them cut off from the rest of the world by a language which they spoke only among themselves [...] This dialect not only served to alienate them from the Gentile world, but even from their own coreligionists in other countries who speak the language of those countries and do not understand Yiddish. Thus the Jew is separated from his fellowJew as well as from his Christian neighbour. [...] Whenever my task seemed hopeless I had only to think of my coreligionists, speechless, and therefore without hope of culture, scattered over the world and hence unable to understand one another, who must needs take their culture from strange and hostile sources - and the thought of all that filled me with renewed energy. Yes, I am convinced that there is no people in the world to whom a world-wide medium of communication like Esperanto might prove so useful as the Jewish people. It would bridge the gap which in all Jewish communities nowadays yawns between the native and the foreigner, the rich and the poor, the cultured and the uncultured ». «Esperanto and Jewish Ideals », op. cit., p. 16, notre trad. 
principe sur lequel il la fonde, et que d'autres traditions qualifient de règle d'or. Mais nous nous en tiendrons principalement à la question de la langue, essentielle puisqu'il était convaincu que «le hillélisme sans la langue ne pourrait d'aucune façon exister, il n'aurait aucun sens et ne constituerait qu'un faux compromis incapable de vie ${ }^{78}$; vie que cette doctrine éthico-religieuse a de toute façon à peine connue, ayant bientôt été remplacée par l'homaranisme. Zamenhof donnera lui-même une explication fort plausible de cet échec : «On m'a très mal compris ; on a dit que je voulais seulement faire de la propagande pour 'mon' espéranto ! C'est pourquoi j'ai dû arrêter... $»^{79}$.

Or il s'agissait de l'inverse : «Ce projet [...] représente en soi à l'état pleinement achevé cette idée à laquelle j'ai consacré toute ma vie ; toute l'affaire de l'espéranto n'est qu'une partie de cette idée commune que je nomme hillélisme ${ }^{80}$; même si l'indispensable langue neutre, indissolublement liée à son vaste projet qui l'englobe, n'en est pas pour autant oubliée :

À la maison chaque hilléliste pourra parler sa langue ou son dialecte lui venant de ses parents et vivre selon les coutumes de sa religion parentale ; mais en tous les lieux et dans toutes les affaires publiques le hilléliste n'imposera jamais à d'autres sa propre langue ou religion mais s'efforcera qu'y règnent seulement une langue et une religion acceptables par tout homme par leur neutralité [neŭtralehomaj]. ${ }^{81}$

Simple élargissement, en fait, de la devise populaire : « un juif à la maison, un homme dans la rue ».

Il s'agissait pour lui de créer, non plus seulement un « pont neutre sur le plan linguistique entre les ethnies ${ }^{82}$, ce que faisait l'espéranto, mais cette fois, «un pont moral par lequel pourraient s'unir fraternellement tous les peuples et religions, sans création d'espèces de

\footnotetext{
${ }^{78}$ Lettre du 15[/28] mai 1901 à Abraham Koffman, dans G. Waringhien, Leteroj de L.L. Zamenhof, op. cit., p. 19, notre trad.

${ }^{79}$ Lettre de Zamenhof à Wilhelm Heller, du 30-6-1914; publiée dans Destino de ludovika dinastio [litt. : Destin de la dynastie ludovicienne], 1907-1917, Tokyo : Eldonejo ludovikito, coll. PVZ, Originalaro 3, 1991, p. 2655, notre trad.

${ }^{80}$ Lettre de Zamenhof à Émile Javal du 24-9-1905, p. 11 ; ms. reprod. dans Aŭtografoj de ludoviko [sic] [Autographes de Louis (= Zamenhof)], [Kyoto ?], Eldonejo ludovikito, coll. PVZ, Kromkajero 4, 1989, p. 141, notre trad., le mot mis par nous en italiques est souligné dans le ms.

${ }^{81}$ Ibid., p. 146, en italiques : mots soulignés par l'auteur, notre trad.

82 «Nefermita letero al s-ro de Beaufront: (de Homarano [L. Zamenhof])», $L^{\prime}$ Espérantiste $\mathrm{n}^{\circ} 101$, juillet 1906, p. 150, notre trad. D'où le titre « Pont de mots » de l'ouvrage d'Esther Schor précédemment cité.
} 
dogmes nouvellement inventés et sans aucun besoin que les peuples rejettent les religions qui sont jusqu'à présent les leurs $»{ }^{83}$.

Et il développe ainsi son idée dans sa lettre à Javal qu'il suppose se demander comment, sans rejeter sa « religion parentale », on peut adopter une religion qui serait acceptable par tous :

Nous pouvons aisément y parvenir (bien sûr chez des personnes pensant de manière logique) par l'introduction du principe, un peu modifié, qu'a un jour donné l'antique rabbin hébreu Hillel (d'où le nom que je lui donne de hillélisme) et qui (sous une forme que j'ai un peu changée) consiste dans le dogme suivant : « La seule loi que nous a donnée la mystérieuse Force morale menant le monde, c'est : 'agis avec les autres comme tu désires que les autres agissent avec toi'; tout [ce qui est] autre dans les religions n'est que coutumes, qu'ont créées les hommes et que nous pouvons et non devons observer $» .^{84}$

La dernière phrase le rapproche de Moses Mendelssohn, qui «soutint que la Tōrah n'est pas une religion révélée, car les vérités rationnelles doivent avoir été mises à la disposition de tout homme ${ }^{85}$. Une fois cette origine humaine admise, et elle l'était par une notable partie des maskilim, le problème était résolu :

Lorsque le nombre des hillélistes (c'est-à-dire ceux qui confessent le principe mentionné) se développera dans le monde, alors il apparaîtra tôt ou tard que tous les hommes n'ont qu'une religion qui est inscrite dans le cœur de chaque homme sous le nom de « conscience », et [qu']ils ne se différencient que par leurs mœurs qui, en tant que création purement humaine, peuvent se perfectionner en permanence et mutuellement se fondre et s'unir. ${ }^{86}$

\section{Dépassement de l'assimilationnisme}

L'idée est, mais en apparence seulement, semblable aux thèses assimilationnistes de l'époque car, d'une part, Zamenhof pensait à des influences réciproques, et donc avec aussi ce sens inattendu :

Cette petite communauté que nous voulons fonder à présent apparaîtra bientôt comme la base ou le levain d'une humanité renaissante et fraternellement unie ;

\footnotetext{
${ }^{83}$ Lettre à A. Michaux, 21-2-1905, dans G. Waringhien, Leteroj de L.-L. Zamenhof, op. cit., p. 107, notre trad.

${ }^{84}$ Lettre à É. Javal, 24-9-1905, p. 16-17, dans Aŭtografoj, op. cit., p. 146-147 (même remarque), notre trad.

${ }^{85}$ Roland Goetschel, « Tōrah », dans Enc. Universalis, 2002, vol. 22, p. 848c.

${ }^{86}$ Lettre de Zamenhof à É. Javal du 24-9-1905, p. 17-18, dans Aŭtografoj, op. cit., p. 147-148, notre trad.
} 
et nous, juifs, devons nous enorgueillir de ce que nous soit échu l'honneur d'apporter au monde cette grande transformation spirituelle, tout comme aux Hébreux de l'Antiquité revint le mérite d'apporter au monde le fondement du monothéisme. ${ }^{87}$

C'est déjà avec cette signification très novatrice qu'il faut donc lire cette phrase d'un mémoire que lui avaient demandé les organisateurs du Congrès international des races, qui s'est tenu du 26 au 29 juillet 1911 à l'Université de Londres : "C'est un point tout à fait indubitable que plus les hommes communiquent sur une base linguistique neutre [...] plus vite ils s'assimileront réciproquement aussi sur le plan religieux $\gg{ }^{88}$.

Et d'autre part, Zamenhof avait totalement revu sa position depuis sa brochure de 1901 où il écrivait :

Résumons $[\ldots]$ l'essentiel de notre projet.

1. Épurer la religion juive $[\ldots]$

2. Créer une langue propre aux juifs ${ }^{89}[\ldots]$

3. Choisir un endroit précis où pourraient converger tous les juifs qui, pour une raison ou une autre, décideraient de quitter leur patrie naturelle afin de vivre entre eux. $^{90}$

Début 1905, en effet, il écrivait déjà : «Il y a eu une époque où je pensais que tous les habitants d'un pays devaient s'efforcer d'avoir une seule religion et de parler une seule langue [...] mais je l'ai très vite rejetée, car je me suis convaincu que l'idée n'était pas bonne ${ }^{91}$; et l'automne de cette même année, mais déjà après le retentissant succès un peu inattendu du premier congrès universel d'espéranto (à Boulogne-sur-

\footnotetext{
87 « Appel aux intellectuels juifs », en annexe à L. L. Zamenhof, Le Hillélisme, op. cit., p. 130-131.

${ }^{88}$ D-ro L. L. Zamenhof, Gentoj kaj Lingvo Internacia: Memuaro verkita [Ethnies et Langue internationale: Mémoire écrit, en nov. 2010] por la Kongreso de Rasoj, reprod. dans Destino, op. cit., p. 2408, notre trad. sur l'original esp. (Trad. anglaise moins fidèle dans G. Spiller (ed.), Papers on Inter-racial problems [Communications sur des problèmes interraciaux], London, P. S. King, 1911, p. 431).

${ }^{89} \mathrm{Ni}$ hébreu ni yiddish et, en principe, laissée au choix d'un futur congrès : soit une « langue neutre déjà existante » (en clair : l'espéranto), soit même encore une «nouvelle langue ». (L. L. Zamenhof, Le Hillélisme, op. cit., p. 107).

${ }^{90}$ Ibid., p. 123. En 1901 il avait renoué, cette fois pour les hillélistes, avec ses idées présionistes en indiquant "par exemple au Canada, aux États-Unis, en Argentine ou ailleurs », ibid., p. 113. Et en 1905, il pensera à « la Suisse qui, n'ayant pas de langue nationale [propre], est le pays le plus neutre de toute l'Europe ». Lettre du 2-10-1905 au pasteur Schneeberger, dans Destino, op. cit., p. 2655. (Nos trad.).

${ }_{91}$ Lettre à A. Michaux, 21-2-1905, op. cit. dans G. Waringhien, Leteroj de L.-L. Zamenhof, p. 109, notre trad.
} 
Mer) : «Le hillélisme a pour but de créer un peuple humain par sa neutralité, et dont les membres ne soient divisés que par des frontières géographiques et politiques [celles qui leur sont imposées], mais non par leurs langues et religions $\gg{ }^{92}$.

Il s'agissait donc là, non plus d'un État (politique) ou d'une nation (juive), ni d'un lieu (Palestine ou autre), mais d'une communauté transnationale tolérante répandue dans le monde entier, fondée sur l'utilisation de l'espéranto et qu'il appelle un peuple car selon lui un groupe ayant une même langue en partage, forme par là un peuple.

Malgré cette dispersion géographique qui objectivement demeurait inchangée, pour lui, grâce à la langue neutre commune, la notion même d'exil semble avoir disparu.

L'assimilation même mutuelle est donc publiquement remise en cause dans le mémoire au Congrès des races car celui-ci ne s'arrêtait pas au constat de la première citation que nous en avons faite mais allait beaucoup plus loin, ce sur quoi l'auteur insiste par la fréquence des mots en italiques :

Ce qui est cause de malheur pour l'humanité, c'est non pas l'existence des ethnies, mais le fait qu'elles s'imposent les unes aux autres d'une manière jusqu'à présent tout à fait inévitable. À chaque fois que je veux entrer en relation avec une personne d'une autre ethnie, il est absolument nécessaire que : soit je lui impose ma langue et mes coutumes, soit il m'impose les siennes. Lorsque disparaîtra cette regrettable nécessité de les imposer, alors disparaîtra la haine interethnique. ${ }^{93}$

C'est l'idée nouvelle, non plus d'homogénéité ethnico-linguistique, et ses inévitables haines de l'autre, voire "épurations», mais de coexistence pacifique dans le respect des différences, et même de cohésion sociale fraternelle d'après ses autres textes.

\section{Épilogue : l'homaranisme}

En 1906, le hillélisme a cédé la place à un projet en fait assez peu différent, à part l'effacement de toute connotation juive car « le hillélisme ne concernait qu'un seul groupe humain alors que l'homaranisme

\footnotetext{
${ }^{92}$ Lettre à É. Javal, 24-9-1905, p. 13, dans Aŭtografoj, op. cit., p. 143, notre trad.

${ }^{93}$ L. L. Zamenhof, Gentoj kaj Lingvo, op. cit., p. 2406-2407, notre trad. (Trad. anglaise moins fidèle dans G. Spiller (ed.), Papers, op. cit., p. 430).
} 
concerne tous les peuples et religions » ${ }^{94}$, et il «exprime plus précisément toute l'essence de notre idée ['homarane' signifie en langue espéranto 'membre de la famille humaine'] $\gg{ }^{95}$.

Il avait donc reçu pour nouvelle définition : «L'homaranisme est la poursuite de la pure homitude et de la justice et égalité interethnique absolue ${ }^{96}$, complétées par une plus positive «fraternité interethnique $\iota^{97}$.

Et voici donc la pierre de touche de l'homaranisme, qui permettait d'y inclure, sans même plus la moindre considération de langue ou de religion, tout homme de bonne volonté actif: l'homarane, écrivait-il, c'est celui qui « du groupe de ceux qui parlent et rêvent [de la fraternité de l'humanité], passe dans le groupe de ceux qui [la] réalisent $»^{98}$.

\section{En guise de conclusion}

Un article, lui aussi sur l'espéranto et l'exil, cite ce frappant amalgame d'un journaliste antisémite: «Vous êtes par vocation l'espéranto errant ${ }^{99}$. Comme nous venons de le voir, la langue avait été inventée précisément pour mettre fin à cet éternel exil.

\footnotetext{
${ }^{94}$ Homaranismo : Plibonigita kaj plikompletigita eldono de Dogmoj de Hilelismo [L'homaranisme : Édition améliorée et complétée des Dogmes du Hillélisme], SaintPetersbourg, 1906 ; reprod. dans Gis la homaranismo, op. cit., p. 1695, notre trad.

95 «Aldono al la 'Dogmoj de Hilelismo'», Ruslanda Esperantisto, II année, n 2 , fév. 1906, notre trad. Même texte, onze ans plus tard, dans la dernière version de Homaranismo (1917, l'année de sa mort).

${ }^{96}$ Ms., de juillet 1906, du discours que Zamenhof prévoyait de faire à l'ouverture du congrès de Genève ; publié sous le titre « Kion Zamenhof ne povis diri en Ĝenevo », La nica literatura revuo, $\mathrm{n}^{\circ} 24,1959$; reprod. dans $\hat{G}$ is la homaranismo, op. cit., p. 1792, notre trad.

${ }_{97}$ Ibid., p. 1798, notre trad.

98 Homarano [pseud. de Zamenhof], «Pri la homaranismo: Respondo al P-ro Dombrovski », Ruslanda Esperantisto, $\mathrm{II}^{\mathrm{e}}$ année, ${ }^{\circ}$ 5, mai 1906 ; reprod. dans $\hat{G}$ is la homaranismo, op. cit., p. 1733-1734, notre trad.

${ }^{99}$ Maurice Gandolphe, «Espéranto », La Liberté, 17-8-1905, p. 1 ; cité par Jean-Claude Lescure, « L'espéranto, ou comment se protéger de l'exil (1900-1930)», op. cit, p. 17.
} 


\section{Zamenhof (1859-1917)}

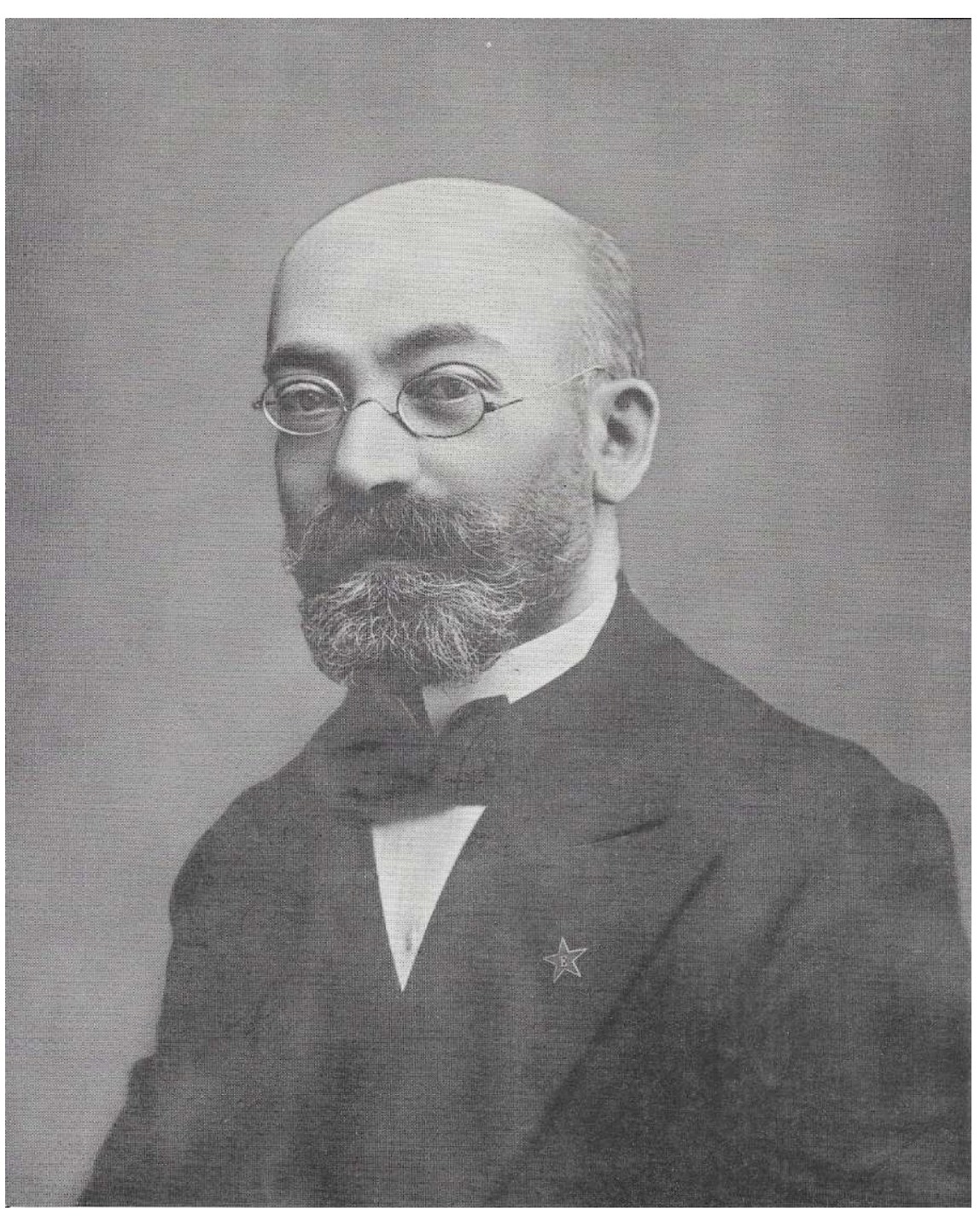

Archives personnelles de l'auteur

De format 21 x $26 \mathrm{~cm}$ et tramée, cette photographie semble avoir été effectuée, peut-être dans les années 1960, à partir de celle reproduite dans le livret du $4^{\mathrm{e}}$ congrès universel d'espéranto qui s'est tenu à Dresde du 16 au 22 août 1908 (Kongresa libro : Eldono de la Kvaro por la Kvara, Dresden, Boden, 1908), soit à partir de l'original, soit plus vraisemblablement à partir d'un exemplaire non identifié du livret, en meilleur état sur ce point que celui conservé dans les archives de l'Association Universelle d'Espéranto (UEA) à Rotterdam, puisque la photo ne comporte pas de rayures (cf. celle, par ailleurs identique, publiée à

https://commons.wikimedia.org/wiki/File:1908-kl-t-zamenhof.jpg). 